\title{
Protocolo de Evaluación Transdiagnóstico para Estrés, Ansiedad y Depresión: Un Análisis con Víctimas Colombianas
}

\author{
Bertha Lucía Avendaño-Prieto ${ }^{1}$ \\ Orcid.org/0000-0002-8136-5380 \\ Ronald Alberto Toro-Tobar ${ }^{*}, 1$ \\ Orcid.org/0000-0001-6061-3499 \\ Nancy Marina Vargas-Espinosa ${ }^{1}$ \\ Orcid.org/0000-0001- 9492-9176 \\ Javier Villalba-Garzón ${ }^{2}$ \\ Orcid.org/0000-0002-5358-2353
}

\author{
${ }^{1}$ Universidad Católica de Colombia, Bogotá, Colombia \\ ${ }^{2}$ Universidad Nacional de Colombia, Bogotá, Colombia
}

\section{Resumen}

Siguiendo el modelo transdiagnóstico, se diseñó un protocolo de evaluación para estrés, ansiedad y depresión. Se realizaron dos estudios. El primero fue de tipo instrumental en el cual se estableció la validez de contenido del protocolo a través del juicio de armonía interjueces, con nueve expertos en evaluación transdiagnóstica, quienes evaluaron los aspectos generales, las variables transdiagnósticas incluidas, las problemáticas clínicas e instrumentos propuestos, mediante la técnica Angoff Modificada. Los coeficientes $r_{\mathrm{wg}}$ oscilaron entre .33 y .97 valores que indicaron acuerdo entre jueces. Los indicadores de severidad e indulgencia permiten inferir que la evaluación de los expertos fue favorable. En el segundo estudio se compararon las puntuaciones medias obtenidas en la aplicación del protocolo a 15 víctimas del conflicto armado y 73 personas sintomáticas. No se encontraron diferencias estadísticamente significativas entre las medias de las variables transdiagnósticas entre los dos grupos los valores $\mathrm{p}$ oscilaron entre .09 y .95 , resultados que permiten inferir que la sensitividad ansiosa, intolerancia a la incertidumbre y afecto positivo-negativo, como variables transdiagnósticas pueden ser identificadas mediante el protocolo, independientemente del tipo de diagnóstico o condición de víctima.

Palabras clave: Estrés, ansiedad, depresión, transdiagnóstico, evaluación, protocolo.

* Dirección para correspondencia: Avenida Caracas \# 46 - 22, Bogotá, Colombia. E-mail: blavendanop@ ucatolica.edu.co, ratoro@ucatolica.edu.co,nmvargas@ucatolica.edu.co y javierandresvillalba@gmail.com Documento derivado del proyecto de investigación institucional titulado: Diseño de un protocolo de evaluación transdiagnóstica para víctimas y no víctimas del conflicto (4110016).

Agradecimientos: Los autores agradecen a la Universidad Católica de Colombia por su apoyo financiero en la realización de esta investigación. 


\title{
Protocolo de Avaliação Transdiagnóstica para o Estresse, Ansiedade e Depressão: Uma Análise com as Vítimas Colombianas
}

\section{Resumo}

O objetivo deste estudo foi descrever as propriedades de um protocolo para a avaliação do estresse, a ansiedade e a depressão, elaborado segundo o modelo transdiagnóstico. Realizaram-se dois estudos. No primeiro (do tipo instrumental) foram procuradas evidências de validade de conteúdo por meio da estimação da concordância entre juízes. Nove especialistas na área de avaliação transdiagnóstica analisaram as características gerais, as problemáticas clínicas, os instrumentos propostos e as variáveis transdiagnósticas contidas no protocolo, por meio da técnica Angoff modificada. Os coeficientes $r_{\text {wg }}$ mostraram uma boa concordância entre os juízes. A análise dos índices de severidade e indulgencia indica que a avaliação dos especialistas foi favorável. No segundo estudo, o protocolo foi aplicado a 15 vítimas do conflito armado e 73 pessoas sintomáticas, e suas pontuações foram comparadas. Não houve diferenças estatisticamente significativas entre os dois grupos quanto às variáveis transdiagnósticas, esses resultados permitem inferir que o protocolo pode ser utilizado para identificar a sensitividade ansiosa, a intolerância à incerteza e o afeto positivo-negativo, como variáveis transdiagnósticas, independentemente do tipo de diagnóstico ou a condição da vítima.

Palavras-chave: Estresse, ansiedade, depressão, transdiagnóstico, protocolo.

\section{Transdiagnostic Evaluation Protocol for Stress, Anxiety and Depression: An Analysis with Colombian Victims}

\begin{abstract}
Two studies were undertaken following the transdiagnostic model, an evaluation protocol for stress, anxiety, and depression was designed. The first one was instrumental, in which the validity of the content of the protocol was established through interjudged harmony judgment, with nine experts in transdiagnostic evaluation, who evaluated the general aspects, the transdiagnostic variables included, the clinical problems, and the instruments proposed, using the modified Angoff technique. The $r_{\mathrm{wg}}$ coefficients fluctuated between .33 and .97 showing inter-judge agreement. The indicators of severity and indulgence allowed us to infer that the evaluation of the experts was favorable. In the second study, the average scores obtained in the application of the protocol were compared to 15 victims of the armed conflict and 73 symptomatic people. There were no statistically significant differences between the means of the transdiagnostic variables between the two groups, $p$-values fluctuated between .09 and .95 , this results allow us to infer that anxiety sensitivity, intolerance to uncertainty and positive-negative affect as transdiagnostic variables can be identified by means of the protocol regardless of the type of diagnosis or condition of victim.
\end{abstract}

Keywords: Stress, anxiety, depression, transdiagnosis, protocol.

El estar expuesto al miedo, al temor, a la muerte, a situaciones conflictivas y a la violencia en general de manera casi permanente, es una experiencia que a través de la historia, se ha demostrado que produce algún tipo de afectación o de secuela psicológica o social, que puede manifestarse de diferentes maneras. El conflicto armado fue considerado uno de los eventos sociales y políticos de gran impacto y el que mayor cantidad de víctimas ha dejado, no solo en Colombia sino en diferentes países que lo han padecido (Vallejo, 2011); antes del proceso de 
negociación y postconflicto, según Pérez-Sales (2010), existían más de dos millones de personas desplazadas debido a la violencia política y más desplazados por razones económicas, que fueron empujados fuera del sistema por una ruptura de la red social, o por la pobreza como consecuencia de la violencia. En el año 2012, año en el que inician los diálogos de paz hasta el 2017 cuando se firma el acuerdo de paz, se documentaron 218094 muertes debidas al conflicto armado (Centro de Memoria Histórica, 2018).

La violencia política en Colombia asociada a fenómenos del conflicto como las acciones de la guerrilla, el narcotráfico, y las fuerzas paramilitares, ha trazado la historia del país desde la década del sesenta, y ante esto, un avance significativo se está viviendo actualmente en lo que respecta a la negociación de paz con un grupo guerrillero (FARC-EP), razón por la cual la atención a las víctimas toma especial interés en el marco del postconflicto, particularmente en verdad y reparación integral (Tobar, 2015).

$\mathrm{Al}$ respecto, los trabajos llevados a cabo con las víctimas, específicamente los relacionados con la memoria histórica, han posibilitado un conocimiento de la oralidad y la comprensión del origen y dinámica de las historias de cada actor del conflicto (Rueda, 2013). Uno de los temas de estudio es la problemática psicológica derivada que ha establecido que los eventos traumáticos deberán ser analizados desde el contexto histórico, el cual permite comprender los elementos transgeneracionales y las respuestas diversas tanto psicológicas como sociales (Borda, Carrillo, Garzón, Ramírez, \& Rodríguez, 2015).

Aunque los estudios sobre las consecuencias se han centrado en aspectos emocionales y traumáticos, se han documentado diferentes problemáticas en torno al conflicto; por ejemplo, la violencia de género y el abuso sexual asociados al desplazamiento e inseguridad (Lemaitre \& Sandvik, 2014), también en síntomas como la paranoia, consumo de alcohol, problemas del estado del ánimo y estrés postraumático (Hewitt et al., 2016).

Andrade (2011), tipificó las patologías en salud mental prevalentes en 36 familias despla- zadas por el conflicto armado en Colombia. Los resultados mostraron la presencia de depresión leve $(20 \%)$, moderada (60\%; Mayor en las mujeres, por cada dos hombres deprimidos hay tres mujeres con esta patología) y depresión grave (30\%; p. 122). Se encontró que el $100 \%$ de la población presentaba indicadores de trastorno de estrés postraumático, con una gravedad extrema del $50 \%$ y moderada del $40 \%$. Los datos indicaron que las secuelas psicológicas del conflicto perduran en las poblaciones desplazadas, constituyéndose en cuadros psicopatológicos comórbidos aun cuando disminuya la inseguridad del entorno.

Restrepo, Yara, Cano y Tavera (2014), realizaron un estudio con una muestra de $80 \mathrm{mi}$ litares del Ejército Nacional de Colombia, cuyo objetivo fue establecer el perfil psicológico en militares, víctimas de minas antipersonas y artefactos explosivos improvisados, más un grupo control, a partir del análisis de cuatro variables emocionales (depresión, ansiedad, ira y estrés). Los resultados mostraron que la ansiedad-estado y rasgo, ira-estado y rasgo presentaron diferencias estadísticamente significativas entre los grupos, aunque en depresión y estrés no se encontraron diferencias.

En un estudio realizado por Aristizábal et al., (2012) se halló que, entre los síntomas psicológicos como consecuencia de la situación del conflicto armado, se encontraban traumatismos psicológicos, tales como temores, angustia, pensamientos obsesivos, actos compulsivos, tristeza, depresión, trastornos en la conducta del sueño, de la conducta sexual, impulsos agresivos, bloqueos y episodios psicóticos.

En poblaciones específicas relacionadas con las víctimas, Ruiz (2014) menciona que, en Colombia, la psicología jurídica ha dado aportes a la valoración del daño asociado con las lesiones psicológicas y no ha sido ajena al estudio de las victimas involucradas en el conflicto armado. Sin embargo, dada la complejidad de la situación, es evidente la necesidad del uso de perspectivas transdiagnósticas que faciliten la identificación de factores de riesgo y factores protectores asociados a la victimización, la prevención de 
la victimización, la aparición de trastornos, así como la reducción de las secuelas producto de la comisión del delito.

El modelo transdiagnóstico según Sandín, Chorot y Valiente (2012) "consiste en entender los trastornos mentales sobre la base de un rango de procesos cognitivos y conductuales etiopatogénicos causales y/o mantenedores de la mayor parte de los trastornos mentales o de grupos consistentes de trastornos mentales" (p. 187), basado en una perspectiva dimensional de los procesos y factores subyacentes a los diferentes trastornos.

Dentro de las aproximaciones al modelo transdiagnóstico, están entre muchos más, los aportes de las escuelas conductistas tradicionales como el condicionamiento clásico y operante para explicar la conducta anormal (Skinner, 1957), los modelos de personalidad basados en neuroticismo, extraversión y psicoticismo (v.g. Eysenck, 1959), los modelos cognitivos del procesamiento de la información aplicado a la psicoterapia (v.g. Beck, 1976), y el modelo de triple vulnerabilidad de Barlow (2002): biológica, psicológica y patológica.

Posteriormente, se han propuesto modelos transdiagnósticos tipo superclase (a diferencia de los categóricos tradicionales tipo DSM-IV-TR) en los cuales los trastornos emocionales y afectivos se comprenden desde una categoría dimensional única que incluye tres subclases: ansiedad-sufrimiento (depresión mayor, distimia, ansiedad generalizada), miedo (pánico, agorafobia, fobia social y específica) y bipolar (bipolar I y II, ciclotimia; Watson, O'Hara \& Stuart, 2008).

Estos modelos han sido derivados en parte de la propuesta de Watson y Clark (1984), en la cual se planteó un continuo de afecto positivo y negativo, definidos en términos de respuesta ante estresores, donde el factor general asociado a la ansiedad es el afecto negativo entendido como la sensibilidad temperamental ante estímulos negativos que se experimenta como emociones negativas tales como miedo, ansiedad, tristeza, culpa, hostilidad, insatisfacción, pesimismo, quejas somáticas, y visión negativa de sí mismo, mientras que con depresión se ha asociado el bajo afecto positivo (bajo entusiasmo, actividad, alerta, energía y escasa participación gratificante; Watson \& Clark, 1984; Watson, Clark \& Tellegen, 1988).

De acuerdo con lo anterior, para el desarrollo de un protocolo de evaluación transdiagnóstico (PET), es necesario tener en cuenta los procesos implicados en los diferentes trastornos emocionales y afectivos (Belloch, 2012). Al respecto, la propuesta de Harvey, Watkins, Mansell y Shafra (2004) presentó cinco dominios claves para la comprensión y el desarrollo de una intervención unificada: atención, memoria, razonamiento, pensamiento y conducta.

Estos dominios han sido propuestos con base en estudios realizados con variables como afecto negativo (AN), intolerancia a la incertidumbre (II) y sensitividad ansiosa (SA), las cuales se definen a continuación.

El afecto negativo (AN) ha sido definido como la sensibilidad temperamental ante estímulos negativos que se experimenta como emociones negativas tales como miedo, ansiedad, tristeza, culpa, hostilidad, insatisfacción, pesimismo, quejas somáticas, y visión negativa de sí mismo, mientras que el afecto positivo (AP), se caracteriza por respuestas como entusiasmo, actividad, alerta, energía y participación gratificante (Watson \& Clark, 1984; Watson et al., 1988).

La intolerancia a la incertidumbre (II) ha sido definida como un sesgo cognitivo en el cual la persona percibe de manera negativa las situaciones en las cuales no tiene certidumbre, se ha reconocido como una variable de vulnerabilidad por la excesiva e incontrolable preocupación Las personas con alta II suelen percibir los eventos futuros como altamente amenazantes, inaceptables y necesitan reducir su posibilidad de ocurrencia, mediante conductas como excesivos chequeos, búsqueda de garantías, e hipervigilancia (Koerner, \& Dugas, 2008).

La sensitividad ansiosa se define como el miedo a las sensaciones relacionadas con la ansiedad (Taylor et al., 2007), constructo de tres dimensiones: somática, social, y cognitiva, relacionado con medidas de estado y rasgo del 
miedo, ansiedad y evitación conductual (Kemper, Lutz, Bähr, Rüddel, \& Hock, 2012; Wilson, \& Hayward, 2006).

El marco del postconflicto en Colombia, afecta toda la población en general, pero específicamente a las víctimas del terrorismo, quienes se visibilizan aún más como resultado de la resolución del conflicto armado sufrido desde la década de los cincuenta, situación que exige preparación para enfrentarla positivamente.

El planteamiento inicial de un PET debe contar con un paso a paso sobre la administración de instrumentos psicométricos y otras herramientas de evaluación sólidas y reconocidas por su evidencia empírica, teniendo en cuenta las variables transdiagnósticas: afecto positivo y negativo, intolerancia a la incertidumbre, y sensitividad ansiosa. Sin embargo, es de reconocer que particularmente para este tipo de población, son pocas las alternativas de evaluación y por lo tanto de intervención disponibles. Por lo anterior, el objetivo de la presente investigación fue elaborar un protocolo de evaluación transdiagnóstica que permita identificar con mayor precisión las variables comunes a los diferentes trastornos (ansiedad, depresión, estrés) frecuentes en las víctimas, aspecto que se constituye en una herramienta de soporte para la posterior intervención.

\section{Estudio 1}

Como parte del análisis psicométrico realizado al protocolo de evaluación transdiagnóstica para estrés, ansiedad y depresión que se diseñó, se estableció la validez de contenido definida como ". . . la relevancia del contenido del test, a la representatividad de los ítems que lo componen, respecto a la totalidad del campo o rasgo objeto de evaluación" (Álvaro, 1997, p. 190), este tipo de validez se puede obtener mediante el concepto de jurados expertos, cuantos más participen mayor validez tiene (Carvajal, Centeno, Watson, Martínez, \& Sanz-Rubiales, 2011),

la consulta a expertos es la vía más usual para apreciar la calidad del contenido, especialmente en ámbitos educativos, aunque cada vez son más empleados los métodos cualitativos basados en la observación directa, las entrevistas o el análisis de archivos. (Prieto \& Delgado, 2010, p. 72)

La técnica Angoff modificada se basa en los juicios de expertos en relación con el tema de evaluación analizado. De acuerdo con la literatura, este método es el más utilizado en diversos procesos que involucran juicios de expertos para procedimientos psicométricos, permite llegar a un consenso de las valoraciones y estimar la precisión de los indicadores revisados de manera numérica (Ricker, 2006). Los datos fueron procesados con el modelo de Rasch y el coeficiente $r_{\text {wg }}$ (Benavente, 2009).

\section{Método}

Tipo investigación. Es un estudio cuantitativo empírico analítico con un diseño no experimental de tipo transversal instrumental (Ato, López, \& Benavente, 2013), el cual buscó establecer la validez de contenido del protocolo de evaluación transdiagnóstica.

Participantes. Los participantes fueron nueve psicólogos clínicos con formación en maestría, todos ellos eran profesores con más de cinco años de experiencia en docencia universitaria en pregrado o postgrado en las asignaturas de psicología clínica, evaluación clínica y psicoterapia.

Instrumentos. Se diseñó un formato para la evaluación por jurados expertos, compuesto por cuatro módulos que evaluaban los aspectos generales, las variables transdiagnósticas, las problemáticas y los instrumentos. La valoración incluyó una escala de uno a diez, siendo diez la máxima calificación, cada experto daba una calificación inicial y una valoración final. El formato tenía también un espacio para agregar comentarios adicionales.

\section{Procedimiento}

Fase 1: construcción del protocolo de evaluación transdiagnóstica siguiendo las recomendaciones para la elaboración de protocolos para el área de la salud.

Fase 2: evaluación de contenido por medio de indicadores de armonía interjueces expertos. 
El procedimiento utilizado fue el método Angoff modificado. Un total de nueve expertos fueron citados a una reunión en la cual cada uno de forma individual evaluaba todos los módulos del protocolo, según una escala de valoración asignada previamente. La calificación se hizo de forma individual pero luego se socializó con los demás expertos, cada quien exponía las razones de su puntaje y las inconsistencias se resolvían in situ. Posterior a la exposición, cada experto de manera individual volvía a puntuar cada elemento del módulo.

Fase 3: Con las calificaciones otorgadas antes y después de la exposición, se estableció el índice de armonía interjueces, calculando las varianzas entre las puntuaciones obtenidas de cada jurado para cada módulo evaluado.

\section{Resultados}

Para realizar el análisis de los datos suministrados por los expertos (su juicio sobre cada uno de los aspectos del protocolo), se utilizó el software Facets (Linacre, 2013) que procesa la información con el Modelo de Rasch. Este análisis proporciona información sobre la consistencia del juicio realizado por los jueces a lo largo de todo el proceso y permite observar la calidad técnica del protocolo. La Severidad / Indulgencia indica el grado de severidad (valor alto, mayor a cero) o indulgencia (valor bajo, menor a cero) de cada calificador. De acuerdo con Linacre (2013) se esperan valores menores o iguales a 2.0. Entre todos los datos que presenta la Tabla 1 , ningún caso es superior a este puntaje, lo que indica que las valoraciones dadas por todos los jueces corresponden con lo esperado por el modelo.

Los ajustes próximo y lejano indican la correspondencia entre la severidad / indulgencia del calificador y el modelo utilizado (Rasch de facetas). Según Linacre (2013) los valores inferiores a 1.00 indican falta de independencia del calificador y los valores superiores a 1.00 indican alta variación en las calificaciones, siendo aceptables valores entre 0.5 y 1.5 . Las valoraciones realizadas por el juez 3 , no se ajustan al modelo.

Tabla 1

Valores Obtenidos por los Psicólogos Clínicos en el Juicio de Expertos

\begin{tabular}{cccc}
\hline Juez & Severidad / Indulgencia & Ajuste Próximo & Ajuste Lejano \\
\hline 3 & .36 & $\mathbf{2 . 7 7}$ & $\mathbf{3 . 2 0}$ \\
1 & -.55 & 1.03 & .96 \\
4 & -.64 & 1.02 & 1.64 \\
5 & -.64 & .56 & .57 \\
7 & -.64 & .50 & .40 \\
2 & -.83 & .54 & .58 \\
6 & -.95 & .43 & .50 \\
8 & -1.17 & .81 & 1.25 \\
9 & -.2 .14 & .68 & $\mathbf{. 3 2}$ \\
\hline
\end{tabular}

Nota. Se resaltan en negrita los indicadores con desajuste. Fuente: Elaboración propia.

Los indicadores de diferencias y acuerdos entre jurados que valoraron el protocolo de evaluación transdiagnóstico para las variables estrés, ansiedad y depresión, en víctimas y no víctimas del conflicto armado se exponen en la Tabla 2.
Los coeficientes de acuerdo de los ítems 4 a 7 fueron inferiores a .30, lo cual indicó baja concordancia. Al eliminar del análisis las valoraciones realizadas por el juez 3 , todos los coeficientes mostraron concordancia. 
Tabla 2

Puntajes Comparados Pre y Post de los Jueces Expertos en los 15 Ítems Evaluados según la Prueba de Rangos con Signos de Wilcoxon y Coeficientes $\boldsymbol{r}_{\mathrm{wg}}$ Pre y Post

\begin{tabular}{lccccc}
\hline Ítems & $\begin{array}{c}\text { Puntaje Z de Prueba } \\
\text { de rangos con signos } \\
\text { de Wilcoxon }\end{array}$ & $\begin{array}{c}\text { Sig. asintótica } \\
\text { (bilateral) }\end{array}$ & $\mathrm{r}_{\text {wg }}$ pre & $\mathrm{r}_{\text {wg }}$ post & $\mathrm{r}_{\text {wg }}$ post sin Juez 3 \\
\hline I1post - I1pre & $-1.638^{\mathrm{a}}$ & .101 & 0.78 & 0.85 & 0.83 \\
I2post - I2pre & $-2.388^{\mathrm{a}}$ & .017 & 0.61 & 0.74 & 0.72 \\
I3post - I3pre & $-1.625^{\mathrm{a}}$ & .104 & 0.67 & 0.79 & 0.79 \\
I4post - I4pre & $-1.289^{\mathrm{a}}$ & .197 & 0.19 & 0.19 & 0.83 \\
I5post - I5pre & $-1.000^{\mathrm{a}}$ & .317 & 0.24 & 0.23 & 0.79 \\
I6post - I6pre & $.000^{\mathrm{b}}$ & 1.000 & 0.14 & 0.14 & 0.94 \\
I7post - I7pre & $.000^{\mathrm{b}}$ & 1.000 & 0.14 & 0.14 & 0.94 \\
I8post - I8pre & $-1.000^{\mathrm{a}}$ & .317 & -0.03 & -0.14 & 0.45 \\
I9post - I9pre & $-1.342^{\mathrm{a}}$ & .180 & 0.64 & 0.40 & 0.33 \\
I10post - I10pre & $.000^{\mathrm{b}}$ & 1.000 & 0.76 & 0.76 & 0.74 \\
I11post - I11pre & $.000^{\mathrm{b}}$ & 1.000 & 0.76 & 0.76 & 0.74 \\
I12post - I12pre & $-1.000^{\mathrm{a}}$ & .317 & 0.60 & 0.64 & 0.80 \\
I13post - I13pre & $-1.000^{\mathrm{a}}$ & .317 & 0.70 & 0.71 & 0.97 \\
I14post - I14pre & $-.447^{\mathrm{c}}$ & .655 & -0.10 & 0.70 & 0.94 \\
I15post - I15pre & $.000^{\mathrm{b}}$ & 1.000 & 0.70 & .70 & 0.92 \\
\hline
\end{tabular}

Notas. Se resaltan en negrita los indicadores con desajuste.

${ }^{a}$ Se basa en rangos positivos.

${ }^{\mathrm{b}}$ La suma de rangos negativos es igual a la suma de rangos positivos.

c Se basa en rangos negativos.

Fuente: Elaboración propia.

Sólo se encontraron diferencias estadísticamente significativas en el ítem 2 , un indicador de los objetivos específicos del protocolo que fueron pertinentes. En la Tabla 2 se observa el nivel de acuerdo de los jurados.

\section{Estudio 2}

En este estudio se realizó una comparación entre muestras sintomáticas y víctimas del conflicto armado colombiano. Se partió de la presunción del modelo transdiagnóstico el cual resalta que existen mecanismos o procesos comunes a diferentes psicopatologías comórbidas (Sandín et al., 2012). Por lo tanto, se asumieron las variables transdiagnósticas como la intolerancia a la incertidumbre, la sensitividad ansiosa y el afecto positivo y negativo como variables transversales a las problemáticas como estrés, ansiedad y depresión independientemente de las condiciones de víctimas o no víctimas del conflicto armado.

\section{Método}

Tipo investigación. Se llevó a cabo un estudio cuantitativo empírico analítico con un diseño no experimental de tipo transversal comparativo (Ato et al., 2013).

Participantes. La muestra estuvo conformada por 88 personas mayores de 18 años, víctimas y no víctimas del conflicto armado, remitidos por entidades gubernamentales y no gubernamentales, con una escolaridad mínima de básica primaria. La muestra de víctimas $(n=15)$ estuvo 
compuesta por seis hombres y nueve mujeres, con una edad media de 44.6 años $(D E=15.32)$, provenientes de diez municipios de los cuales fueron desplazados forzosamente por los grupos armados ilegales de Colombia, un $33.3 \%$ de ellos fueron señalados de pertenecer a un grupo armado, un $20 \%$ por ser líderes comunitarios, el $46.7 \%$ vivieron el asesinato del proveedor económico del hogar y posterior desintegración familiar, además el $60 \%$ presenció masacres de forma directa e indirecta, tres de ellos víctimas de tortura $(20 \%)$, un $66.7 \%$ tuvieron despojo posterior de sus tierras.

La muestra de referencia clínica se obtuvo por medio de los puntos de corte de los instrumentos usados en el estudio, siguiendo las recomendaciones de los autores en cuanto a la estimación de los puntajes como sugerentes de posible patología. Las muestras quedaron distribuidas así: grupo de ansiosos y depresivos $(n=$ 15; $\mathrm{BAI}>25$, BDI-II > 20; edad $M=29.6, D E$ $=9.88$ años; $73.3 \%$ mujeres, $26.7 \%$ hombres), grupo de ansiosos y no depresivos $(n=22$; BAI $>25$, BDI-II $<20$; edad $M=29.14, D E=10.99$ años; $59.1 \%$ mujeres, $40.9 \%$ hombres), grupo depresivos y no ansiosos $(n=13$; BDI-II $>$ 21 , BAI $<25$; edad $M=28.38, D E=5.65$ años; $76.9 \%$ mujeres, $23.1 \%$ hombres), grupo estrés no ansiosos y no depresivos ( $n=25$; EPGE $>40$, BAI $<17$, BDI-II $<20$; edad $M=25.36, D E=$ 5.47 años; $80 \%$ mujeres, $20 \%$ hombres).

\section{Instrumentos}

Escalas de afecto positivo y negativo-PANAS. El PANAS un cuestionario diseñado para evaluar el afecto positivo y negativo como estado y rasgo, por medio de 40 ítems presentados en formato Likert 11 (muy poco o nada), 5 (extremadamente)]. Entre sus propiedades psicométricas reportadas en su adaptación al idioma español fueron favorables índices de consistencia interna tanto para el afecto positivo $(\alpha=.85 \mathrm{y}$ .90) como el afecto negativo $(\alpha=.81$ y .85), se reportaron también significativas correlaciones positivas con ansiedad $(r=.32)$ y depresión $(r=$ .55; Robles \& Páez, 2003).

Anxiety Sensivity Index-ASI3. Este cuestionario fue diseñado para evaluar las respues- tas asociadas a la experiencia subjetiva y física de las manifestaciones de miedo-ansiedad en tres subescalas: sensibilidad a la ansiedad física, cognitiva y social. Se compone de 18 ítems, seis para cada subescala, en formato tipo Likert [Nada o casi nada (0) y Muchísimo (4)]. Entre sus propiedades psicométricas con muestras españolas, presentó una consistencia interna favorable para cada uno de sus factores $(\alpha=.89, .90 \mathrm{y}$ .85; Sandín, Valiente, Chorot, \& Santed, 2007).

Escala de intolerancia hacia la incertidumbre -EII. Esta escala fue diseñada para evaluar las dimensiones de incertidumbre emocional, en términos de preocupación o ansiedad frente a lo incierto. La EII se compone de 27 ítems cada uno con cinco opciones de respuesta $(1=$ Nada característico de mí, y $5=$ Extremadamente característico de mí). Entre sus propiedades psicométricas en su adaptación al idioma español se encontró un elevado coeficiente de consistencia interna $(\alpha=.91)$ y estabilidad test-retest $r=.78$ (González, Cubas, Rovella, \& Darías, 2006).

Escala de percepción global del estrés -EPGE. Esta escala fue diseñada para evaluar el nivel de estrés percibido durante el último mes. Presenta un formato de 14 ítems con opciones de respuesta tipo Likert de cinco puntos $(0=$ nunca, $1=$ casi nunca, $2=$ de vez en cuando, $3=$ a menudo, $4=$ muy a menudo). Sus autores reportan que una puntuación directa indica mayor nivel de estrés percibido, con un punto de corte de 30. Entre sus propiedades presentó una favorable validez concurrente con el STAI $(r=.459, p$ $=.001)$, y adecuada consistencia interna $(\alpha=.79)$ en una muestra chilena (Tapia, Cruz, Gallardo, \& Dasso, 2007).

Inventario de Ansiedad de Beck -BAI. Este inventario está compuesto de 21 ítems de cuatro opciones de respuesta que deben ir acordes a la intensidad del síntoma ansioso vivenciado en la última semana ( 0 : en absoluto, 1: levemente, no me molesta mucho, 2: moderadamente, fue muy desagradable, pero podía soportarlo y 3 : severamente, casi no podía soportarlo). Entre sus propiedades psicométricas con muestras españolas, presentó una adecuada consistencia interna ( $\alpha$ $=.84) \mathrm{y}$ confiabilidad test-retest $(r=.75$; Sanz, Garcia-Vera, \& Fortun, 2012). 
Inventario de depresión de Beck en español, segunda edición -BDI-II. Este inventario contiene 21 ítems con cuatro opciones que varían según la intensidad del síntoma depresivo, entre ellos están la tristeza, llanto, pérdida de placer, sentimientos de fracaso y de culpa, pensamientos o deseos de suicidio, pesimismo, entre otros. La puntuación total se obtiene con una sumatoria total que va entre 0 y 63 puntos, con un punto de corte de 19. Este instrumento presentó una elevada consistencia interna $(\alpha=.94$ y $\alpha=.88$ en pacientes diagnósticados previamente con depresión mayor) en muestras argentinas (Brenlla \& Rodríguez, 2006).

Procedimiento. Los participantes fueron invitados al estudio mediante convocatoria abierta y voluntaria, los cuales se sometieron a una verificación previa de los criterios de inclusión y exclusión. Posteriormente, se les explicó el objetivo general de la investigación y se solicitó firmar el consentimiento informado en el que fueron expuestas las consideraciones éticas del estudio. A continuación, diligenciaron la batería de instrumentos autoaplicados con una duración aproximada de 20 minutos. Se les indicó que todas las inquietudes relacionadas con los resultados podrían ser solicitadas vía email al autor principal de la investigación.

Los análisis de los resultados se llevaron a cabo mediante el software SPSS22 con el cual se obtuvieron las medidas de tendencia central como media, desviación estándar, y dispersión como el rango, asimetría y curtosis en todas las variables transdiagnósticas y sintomáticas. Luego se llevaron a cabo pruebas de normalidad con la prueba Shapiro-Wilk $(p>.05)$, recomendada para muestras menores a 50 casos. Finalmente, se comprobaron las diferencias de medias para muestras independientes con la $t$ de Student con un $95 \%$ de intervalo de confianza.

\section{Resultados}

Las víctimas del conflicto armado obtuvieron altas puntuaciones en las medidas sintomáticas de ansiedad y estrés $(M=19.20, D E=13.69$, y $M=48, D E=11.03)$, y menor en depresión $(M=12.27, D E=9.24)$. En las variables trans- diagnósticas medidas en las víctimas, no se observaron diferencias en las puntuaciones totales con respecto a los grupos sintomáticos (SA: $M=$ 21.13, $D E=15.37$; II: $M=63.33, D E=25.19$; y APN: $M=55.80, D E=7.19$ ). Las diferencias entre grupos sintomáticos y víctimas del conflicto armado se observan en la Tabla 1 por cada variable transdiagnóstica según el grupo sintomático.

\section{Discusión}

Se obtiene evidencia para la hipótesis planteada en este Estudio 2. Los resultados obtenidos en las pruebas de comparación de medias que no fueron estadísticamente significativas, permiten aseverar que las variables transdiagnósticas como la II, la SA y el APN como variables transversales a las problemáticas como estrés, ansiedad y depresión, se identifican de manera independiente de las condiciones de pertenecer a los grupos de víctimas o no víctimas del conflicto armado.

Estos resultados favorecen el desarrollo de herramientas de evaluación e intervención transversales e independientes de las patologías comórbidas e incluso las condiciones premórbidas, aunque es necesario ampliar el estudio a estas condiciones. Es así que los síntomas depresivos, ansiosos y de estrés, pueden ser estudiados a partir de las variables transdiagnósticas SA, II y APN, tal como Talkovsky y Norton (2016), sugieren que una persona con elevado afecto negativo, puede presentar a su vez alta II y SA, además de un incremento en las conductas de evitación, como variables transdiagnósticas complementarias que facilitarían la comprensión de los trastornos ansiosos y otras patologías (Carleton et al., 2012), aspecto clave en la comprensión y tratamiento de los cuadros clínicos comórbidos basados en el modelo transdiagnóstico (Brown, Meiser-Stedman, Woods, \& Lester, 2016).

En conclusión, el presente estudio aportó evidencia empírica de la capacidad convergente del protocolo diseñado para evaluar las variables transdiagnósticas SA, II y APN en víctimas del conflicto armado y personas con problemas de estrés, ansiedad y depresión. 
Tabla 3

Prueba de Diferencias de Medias entre Grupos Sintomáticos y Víctimas del Conflicto

\begin{tabular}{|c|c|c|c|c|c|c|}
\hline \multirow[t]{2}{*}{ Grupos } & \multirow{2}{*}{$\begin{array}{c}\begin{array}{c}\text { Variables } \\
\text { transdiagnósticas }\end{array} \\
\text { SA } \\
(M=29.14, D E=10.92)\end{array}$} & \multirow{2}{*}{$\begin{array}{c}t \\
-1.739\end{array}$} & \multirow{2}{*}{$\begin{array}{c}p \\
.095\end{array}$} & \multirow{2}{*}{$\begin{array}{c}\begin{array}{c}\text { Diferencia } \\
\text { de medias }\end{array} \\
8.00\end{array}$} & \multicolumn{2}{|c|}{$\begin{array}{l}95 \% \text { de intervalo de } \\
\text { confianza de la diferencia }\end{array}$} \\
\hline & & & & & -17.512 & 1.506 \\
\hline \multirow[t]{3}{*}{$\begin{array}{l}\text { Ansiedad } \\
\text { y víctimas }\end{array}$} & $\begin{array}{c}\text { II } \\
(M=66.05, D E=17.18)\end{array}$ & -.363 & .720 & 2.712 & -18.165 & 12.741 \\
\hline & $\begin{array}{c}\text { APN } \\
(M=58.50, D E=9.03)\end{array}$ & -1.332 & .192 & 6.373 & -16.093 & 3.347 \\
\hline & $\begin{array}{c}\text { SA } \\
(M=24.08, D E=12.10)\end{array}$ & -.311 & .758 & $-1,617$ & $-17,512$ & 1.506 \\
\hline \multirow[t]{3}{*}{$\begin{array}{l}\text { Depresión } \\
\text { y víctimas }\end{array}$} & $\begin{array}{c}\text { II } \\
(M=79.08, D E=19.20)\end{array}$ & -1.582 & .127 & $-12,833$ & $-29,568$ & 3.902 \\
\hline & $\begin{array}{c}\mathrm{APN} \\
(M=54.38, D E=6.34)\end{array}$ & -.380 & .707 & $-1,850$ & $-11,869$ & 8.169 \\
\hline & $\begin{array}{c}\mathrm{SA} \\
(M=33.27, D E=15.63)\end{array}$ & $-1,843$ & .076 & $-9,795$ & $-20,702$ & 1,112 \\
\hline \multirow[t]{3}{*}{$\begin{array}{l}\text { Ansiedad-depresión } \\
\text { y víctimas }\end{array}$} & $\begin{array}{c}\text { II } \\
(M=66.93, D E=23.31)\end{array}$ &,- 061 & .952 &,- 524 & $-18,089$ & 17.041 \\
\hline & $\begin{array}{c}\text { APN } \\
(M=59.07, D E=8.11)\end{array}$ & $-1,631$ & .115 & $-7,386$ & $-16,679$ & 1.907 \\
\hline & $\begin{array}{c}\mathrm{SA} \\
(M=15.80, D E=7.97)\end{array}$ & 1.247 & .228 & 5.333 & -3.633 & 14.299 \\
\hline \multirow[t]{2}{*}{ Estrés y víctimas } & $\begin{array}{c}\text { II } \\
(M=60.12, D E=13.83)\end{array}$ & .455 & .654 & 3.213 & $-11,572$ & 17.999 \\
\hline & $\begin{array}{c}\mathrm{APN} \\
(M=56.60, D E=7.53)\end{array}$ & -.676 & .504 & 3.040 & -12.172 & 6.092 \\
\hline
\end{tabular}

Nota. M (Media); DE (Desviación Estándar); SA (Sensitividad Ansiosa); II (Intolerancia a la Incertidumbre); APN (Afecto Positivo y Negativo).

Fuente: Elaboración propia.

\section{Discusión General}

El propósito del presente estudio fue desarrollar y validar un protocolo de evaluación transdiagnóstica como una herramienta que permita mejorar los procesos de evaluación en casos de ansiedad, estrés y depresión, según las variables transdiagnósticas afecto positivo y negativo, sensitividad ansiosa e intolerancia a la incertidumbre. De acuerdo con los resultados obtenidos, el módulo de evaluación y las sesiones que lo componen, evidencian que hubo un acuerdo favorable entre los jueces sin diferencias significativamente relevantes, lo que permite afirmar que el objetivo trazado se cumplió y el protocolo elaborado puede ser implementado en fase de pilotaje.

No encontrar diferencias estadísticamente significativas entre los grupos sintomáticos y las víctimas del conflicto, permite inferir que las variables SA, II y APN, pueden ser identificadas mediante el protocolo independientemente del 
tipo de diagnóstico o condición de víctima. Se puede afirmar que las víctimas presentan un perfil que se asemeja al clínico, aspecto que resalta la importancia de enfocar de forma transdiagnóstica, los procesos de intervención en este tipo de población.

Teniendo en cuenta que el modelo transdiagnóstico ha demostrado proveer respuestas más efectivas a trastornos específicos que presentan altas tasas de comorbilidad y covariación (Belloch, 2012), cada una de las sesiones que componen el módulo de evaluación, se encaminó a analizar la problemática del consultante teniendo como base las variables transdiagnósticas en los casos de depresión, ansiedad y/o estrés.

De esta forma, el protocolo permite identificar los mantenedores específicos que ofrecen al terapeuta una alternativa para la implementación de un tratamiento ajustado a las necesidades específicas de los consultantes, es así que de acuerdo con los datos obtenidos en la evaluación por jueces, tanto las sesiones como el módulo de evaluación transdiagnóstica presentan un adecuado nivel de acuerdo, lo que indica una buena estructura para su posterior etapa de evaluación de efectividad.

Asumir la utilidad clínica de un protocolo transdiagnóstica con víctimas del conflicto armado colombiano, permitirá reducir con mayor celeridad y precisión los síntomas de trastornos de ansiedad y trastornos del estado del ánimo, tal como reportan autores como Romero-Acosta, Ramirez-Giraldo, Hernandez-Bustamante y Porras-Mendoza (2017), sobre las secuelas derivadas del elevado estrés debido a la presencia de grupos paramilitares en la zona de los Montes de María, lugar con una elevada actividad armada actualmente, ya que estas comunidades se han expuesto a permanentes violaciones de los derechos humanos y los derechos internacionales humanitarios, como "masacre, desplazamiento, constreñimiento, extorsiones, amenazas y violencia sexual" (p. 11).

Por otra parte, según Harvey et al. (2004), se puede afirmar que dentro del modelo transdiagnóstico existen variaciones que buscan explicar la conducta humana, en un rango limitado de conductas con múltiples procesos causales, así mismo, se encuentran los que hacen referencia a procesos cognitivos que subyacen a diferentes trastornos, y otros que son procesos simples pero universales para la mayoría de problemáticas conductuales y finalmente, procesos universales presentes para la mayoría de trastornos con o sin una teoría integrativa. Por esta razón, el modelo transdiagnóstico puede incluir diferentes modelos que darían lugar a diferencias en las opiniones de los jueces, aspecto que explicaría las diferencias encontradas en los puntajes iniciales del Angoff.

Se considera pertinente iniciar un proceso de aplicación del protocolo de forma que se pueda identificar la eficacia terapéutica del mismo, por medio de la validez ecológica y el análisis de eficacia del protocolo, con el fin de identificar su impacto a nivel terapéutico en términos de utilidad y eficacia.

\section{Contribuciones de los autores}

Contribución sustancial en el concepto y diseño del estudio: Bertha Lucía AvendañoPrieto, Ronald Alberto Toro Tobar, Nancy Vargas Espinosa.

Contribución a la recolección de datos: Ronald Alberto Toro Tobar.

Contribución al análisis e interpretación de datos: Bertha Lucía Avendaño-Prieto, Ronald Alberto Toro Tobar, Javier Villalba-Garzón.

Contribución a la preparación del manuscrito: Bertha Lucía Avendaño-Prieto, Ronald Alberto Toro Tobar.

Contribución a la revisión crítica, agregando contenido intelectual: Bertha Lucía Avendaño-Prieto, Ronald Alberto Toro Tobar.

\section{Conflictos de interes}

Los autores declaran que no tienen conflicto de intereses con respecto a la publicación de este manuscrito.

\section{Referencias}

Álvaro, M. (1997). Psychometric principles of Psychological Assessment. In G. Buela-Casal \& J. C. Sierra (1997), Psychological Assessment Manual. Fundamentals, Techniques and Applications (pp. 173-192). Madrid: 21st Century. 
Andrade, J. A. (2011). Psychopathological effects of the Colombian Armed Conflict on families in a situation of forced displacement resettled in the municipality of Cairo in 2008. Human Sciences Scientific Journal, 7(20), 111-114. Retrieved from http://www.redalyc.org/articulo. oa?id=70922149005

Aristizábal, E., Palacio, J., Madariaga, C., Osman, H., Parra, L. H, Rodríguez, J., \& López, G. (2012). Symptoms and psychic traumatism in victims and victimizers of the armed conflict in the Colombian Caribbean. Psychology in the Caribbean (Colombia), 29(1), 123-152.

Ato, M., López, J. J., \& Benavente, A. (2013). A Classification System of Research Designs in Psychology. Annals of Psychology, 29(3), 10381059. doi: 10.6018/analesps.29.3.178511

Barlow, D. H. (2002). Anxiety and its disorders: The nature and treatment of anxiety and panic $\left(2^{\text {nd }}\right.$ Ed.). New York: Guilford Press.

Beck, A. T. (1976). Cognitive therapy and the emotional disorders. New York: International Universities Press.

Belloch, A. (2012). Proposals for a transdiagnostic approach to mental and behavioral disorders: Evidence, usefulness and limitations. Journal of Psychopathology and Clinical Psychology, 17(3), 295-311.

Benavente, A. P. (2009). Measures of agreement and bias between judges (Unpublished doctoral dissertation). University of Murcia, Department of Basic Psychology and Methodology, Murcia, Spain. Retrieved from http://www.tdx.cat/ bitstream/handle/10803/112752/TAPBR.pdf;jse ssionid $=5$ D 7372847B8C766D5D16ACE3447B F0F2?sequence $=1$

Borda, J. P., Carrillo, J. O., Garzón, D. F., Ramírez, M. P., \& Rodríguez, N. (2015). Historical trauma. systematic review of a different approach to the armed conflict. Colombian Journal of Psychiatry, 44(1), 41-49. doi: 10.1016/j.rcp.2014.09.005

Brenlla, M., \& Rodríguez, C. (2006). Beck BDIII Depression Inventory Manual Argentine Adaptation. Buenos Aires: Paidós.

Brown, H. M., Meiser-Stedman, R., Woods, H., \& Lester, K. J. (2016). Cognitive vulnerabilities for depression and anxiety in childhood: Speci- ficity of anxiety sensitivity and rumination. $B e-$ havioural and Cognitive Psychotherapy, 44(1), 30-42. doi: $10.1017 / \mathrm{S} 1352465814000472$

Carleton, R. N., Mulvogue, M. K., Thibodeau, M. A., McCabe, R. E., Antony, M. M., \& Asmundson, G. J. (2012). Increasingly certain about uncertainty: Intolerance of uncertainty across anxiety and depression. Journal of Anxiety Disorders, 26(3), 468-479. doi: 10.1016/j.janxdis.2012.01.011

Carvajal, A., Centeno, C., Watson, R., Martínez, M., \& Sanz-Rubiales, A. (2011). How to validate a health measurement tool. Directory of the Navarra Health System, 34(1), 63-72.

Centro de Memoria Histórica. (2018). Statistics on the armed conflict in Colombia. Retrieved from http://www.centrodememoriahistorica.gov.co/ micrositios/informeGeneral/estadisticas.html

Eysenck, H. J. (1959). Maudsley Personality Inventory. London: University of London.

González, R. M., Cubas, L. R., Rovella, A. T., \& Darías, H. M. (2006). Spanish adaptation of the Intolerance of Uncertainty Scale: Cognitive processes, anxiety and depression. Psychology and Health, 16, 219-233.

Harvey, A. G., Watkins, E. R., Mansell, W., \& Shafran, R. (2004). Cognitive behavioral processes across psychological disorders: A transdiagnostic approach to research and treatment. Oxford, UK: Oxford University Press.

Hewitt, N., Juárez, F., Parada, A. J., Guerrero, J., Romero, Y. M., Salgado, A. M., \& Vargas, M. V. (2016). Psychological affectations, coping strategies and levels of resilience in adults exposed to the armed conflict in Colombia. Colombian Journal of Psychology, 25(1), 125140. doi: 10.15446/rcp.v25n1.49966

Kemper, C. J., Lutz, J., Bähr, T., Rüddel, H., \& Hock, M. (2012). Construct validity of the Anxiety Sensitivity Index-3 in clinical samples. Assessment, 19(1), 89-100. doi: $10.1177 / 1073191111429389$

Koerner, N., \& Dugas, M. J. (2008). An investigation of appraisals in individuals vulnerable to excessive worry: The role of intolerance of uncertainty. Cognitive Therapy and Research, 32(5), 619-638. doi: 10.1007/s10608-0079125-2 
Lemaitre, J., \& Sandvik, K. B. (2014). Beyond sexual violence in transitional justice: Political insecurity as a gendered harm. Feminist Legal Studies, 22(3), 243-261. doi: 10.1007/s10691014-9274-0

Linacre, J. M. (2013). Winsteps ${ }^{\circledR}$ (Version 3.90.1) [Computer Software]. Beaverton, Oregon: Winsteps.com. Retrieved from http://www. winsteps.com/

Pérez-Sales, P. (2010). Identity and trauma in adolescents within the context of political violence: A psychosocial and communitarian view. Clinical Social Work Journal, 38(4), 408417. doi: 10.1007/s10615-010-0262-9

Prieto, G., \& Delgado, A. R. (2010). Reliability and validity. Papers on Psychology, 31(1), 67-74. Retrieved from http://www.papelesdelpsicologo.es/pdf/1797.pdf

Restrepo, J. E., Yara, E. A., Cano, J., \& Tavera, L. N. (2014). Emotional profile of a group of Colombian military victims of anti-personnel landmines or improvised explosive devices. Colombian Journal of Psychiatry, 43(2), 87-95. doi: 10.1016/j.rcp.2014.02.004

Ricker, K. (2006). Setting Cut-Scores: A critical review of the Angoff and modified Angoff methods. The Alberta Journal of Educational Research, 52(1), 53-64.

Robles, R., \& Páez, F. (2003). Study on the translation into Spanish and the Psychometric Properties of the Positive and Negative Affect Schedule (PANAS). Mental Health, 26(1), 69-75.

Romero-Acosta, K., Ramirez-Giraldo, A., Hernandez-Bustamante, O., \& Porras-Mendoza, E. (2017). Mental health status of victims of the armed conflict in Chengue. Psychology of the Caribbean, 34(1), 1-20. Retrieved from http:// rcientificas.uninorte.edu.co/index.php/psicologia/article/viewFile/7691/9775

Rueda, J. F. (2013). "Reasoned Historical Memory" An inclusive proposal for victims of the internal Colombian armed conflict. Historelo. Journal on Regional and Local History, 5(10), 15-52.

Ruiz, J. I. (2014). Social Psychology and Justice. Bogotá: National University of Colombia.

Sandín, B., Chorot, P., \& Valiente, R. M. (2012). Transdiagnosis: New frontier in clinical psychology. Journal of Psychopathology and Clinical Psychology, 17(3), 185-203.

Sandín, B., Valiente, R. M., Chorot, P., \& Santed, M. A. (2007). ASI-3: New Scale to Evaluate Anxiety Sensitivity. Journal of Psychopathology and Clinical Psychology, 12(2), 91-104. Retrieved from http://www.aepcp.net/rppc.php?id=209

Sanz, J., García-Vera, M., \& Fortun, M. (2012). The "Beck Anxiety Inventory" (BAI): Psychometric properties of the Spanish version in patients with psychological disorders Behavioral Psychology/ Psicología Conductual, 20(3), 563-583.

Skinner, B. F. (1957). Verbal behavior. Acton, MA: Copley.

Talkovsky, A. M., \& Norton, P. J. (2016). Intolerance of uncertainty and transdiagnostic group cognitive behavioral therapy for anxiety. Journal of Anxiety Disorders, 41, 108-114. doi: 10.1016/j. janxdis.2016.05.002

Tapia, D., Cruz, C., Gallardo, I., \& Dasso, M. (2007). Adaptation of the Perceived Stress Scale (PSS) in Low-Income Adult Students in Chile. Psychiatry and Mental Health, 24(1), 109-119.

Taylor, S., Zvolensky, M. J., Cox, B. J., Deacon, B., Heimberg, R. G., Ledley, D. R. ...Cárdenas, S. J. (2007). Robust dimensions of anxiety sensitivity: Development and initial validation of the Anxiety Sensitivity Index-3 (ASI-3). Psychological Assessment, 19(2), 176-188. doi: 10.1037/1040-3590.19.2.176

Tobar, J. A. (2015). Political violence and dirty war in Colombia. Report from a victim of the Colombian conflict with regard to the negotiations in Havana. Memory and Society, 19(38), 11-24. doi: 10.11144/Javeriana.mys1938.vpgs

Vallejo, G. D. (2011). Quality of life in a population displaced by the internal conflict in Colombia. Amérique Latine Histoire et Mémoire. Les Cahiers ALHIM, 21. Retrieved from http:// alhim.revues.org/3822

Watson, D., \& Clark, L. A. (1984). Negative affectivity: The disposition to experience aversive emotional states. Psychological Bulletin, 96, 465-490. doi: 10.1037/0033-2909.96.3.465

Watson, D., Clark, L. A., \& Tellegen, A. (1988). Cross-cultural convergence in the structure of 
mood: A Japanese replication and a comparison with U.S. findings. Journal of Personality and Social Psychology, 47(1), 127-144. doi: 10.1037/0022-3514.47.1.127

Watson, D., O’Hara, M. W., \& Stuart, S. (2008). Hierarchical structures of affect and psychopathology, and their implications for the classification of emotional disorders. Depression and Anxiety, 25, 282-288. doi: 10.1002/da.20496
Wilson, K. A., \& Hayward, C. (2006). Unique contributions of anxiety sensitivity to avoidance: A prospective study in adolescents. Behaviour Research and Therapy, 44(4), 601-609. doi: 10.1016/j.brat.2005.04.005

Recebido: 05/12/2016

$1^{a}$ revisão: 04/01/2018

Aceite final: 05/01/2018

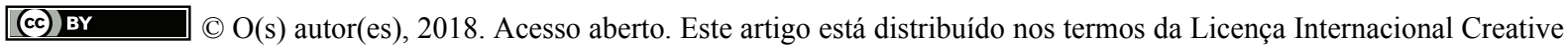
Commons Atribuição 4.0 (http://creativecommons.org/licenses/by/4.0/), que permite o uso, distribuição e reprodução sem restrições em qualquer meio, desde que você dê crédito apropriado ao(s) autor(es) original(ais) e à fonte, fornecer um link para a licença Creative Commons e indicar se as alterações foram feitas. 